Supporting information

\title{
Unique Self-Reinforcing and Rapid Self-Healing Polyampholyte Hydrogels with pH-Induced Shape Memory Effect
}

Yuancheng Zhang, ${ }^{1,2}$ Qiqian Hu, ${ }^{1}$ Shurui Yang, ${ }^{1}$ Tao Wang* ${ }^{* 1,3}$ Weixiang Sun*, ${ }^{1,3}$ Zhen Tong ${ }^{1}$

${ }^{1}$ Research Institute of Materials Science, South China University of Technology, Guangzhou 510640, China.

${ }^{2}$ Liming Research \& Design Institute of Chemical Industry Co., Ltd., Luoyang, 471000, China. ${ }^{3}$ Guangdong Provincial Key Enterprise Laboratory of Novel Polyamide 6 Functional Fiber Materials Research and Application, Jiangmen 529100, China.

*fetwang@scut.edu.cn (T. Wang), mswxsun@scut.edu.cn (W. Sun) 


\section{The viscoelastic-strain-hardening model of the hydrogel}

The stress-strain curves of the hydrogels from uniaxial tensile tests at constant engineering strain rate are the combination of viscoelasticity and strain-hardening. To show this, a simplest constitutive model that consists solely of these two behaviors is implemented to fit the data. In general, the total stress tensor $\boldsymbol{\sigma}$ of an isotropic, homogeneous and incompressible body can be written into the form $\boldsymbol{\sigma}=-p \mathbf{I}+\boldsymbol{\tau}$, where $p$ is proportional to the hydrostatic pressure; $\mathbf{I}$ is the identity tensor. The extra stress tensor $\tau$ is related to the constitutive model of this body; here it is the sum of the viscoelastic part $\boldsymbol{\tau}_{\mathrm{v}}$ and the strain-hardening part $\boldsymbol{\tau}_{\mathrm{s}}$,

$$
\tau=\tau_{\mathrm{v}}+\boldsymbol{\tau}_{\mathrm{s}}
$$

The viscoelastic part is an upper-convected Maxwell (UCM) model by which the Maxwell model can be written into a frame-invariant tensorial form,

$$
\lambda \boldsymbol{\tau}_{\mathrm{v}}^{\nabla}+\boldsymbol{\tau}_{\mathrm{v}}=2 \eta \mathbf{D}
$$

where $\stackrel{\nabla}{\boldsymbol{\tau}_{\mathrm{v}}}$ denotes the upper-convected derivative of $\boldsymbol{\tau}_{\mathrm{v}} ; \mathbf{D}$ is the rate-of-strain tensor; $\lambda$ is the relaxation time of viscoelasticity; $\eta$ is the viscosity of viscoelasticity. The strain-hardening part is a Gent model,

$$
\boldsymbol{\tau}_{\mathrm{s}}=\frac{E J_{\mathrm{m}}}{3\left(J_{\mathrm{m}}-I_{\mathbf{B}}\right)} \mathbf{B}
$$

where $\mathbf{B}$ is the left Cauchy-Green strain tensor; $I_{\mathbf{B}}$ is the first invariant of $\mathbf{B} ; J_{\mathrm{m}}$ is a limiting parameter so that the stress diverges as $I_{\mathbf{B}}$ is approaches $J_{\mathrm{m}} ; E$ acts as the modulus for the strainhardening part.

Under uniaxial tension at constant engineering strain rate $\dot{\varepsilon}$, the principal stretch ratio $\alpha$ is related to the tensile strain $\varepsilon \equiv \dot{\varepsilon} t$ by $\alpha=\varepsilon+1$, and $I_{\mathbf{B}}$ is related to the $\alpha$ by $I_{\mathbf{B}}=\alpha^{2}+2 \alpha^{-1}-3$. 
The tensile true stress $\sigma_{\mathrm{t}}$ is the normal stress difference of the total stress tensor, $\sigma_{\mathrm{t}}=\sigma_{11}-\sigma_{22}=$ $\tau_{11}-\tau_{22}$, where $\sigma_{i j}$ and $\tau_{i j}$ are the components of the total and extra stress tensors $\boldsymbol{\sigma}$ and $\boldsymbol{\tau}$, respectively. The solution to eq. (2) for uniaxial tension at constant $\dot{\varepsilon}$ is given by

$$
\begin{aligned}
& \tau_{\mathrm{v} 11}= \begin{cases}\frac{2 \eta}{\lambda} \exp \left(\frac{1+\dot{\varepsilon} t}{\dot{\varepsilon}} \frac{2 \lambda-1}{\lambda}\right)\left[E i\left(-\frac{1+\dot{\varepsilon} t}{\varepsilon} \frac{2 \lambda-1}{\lambda}\right)-E i\left(-\frac{2 \lambda-1}{\dot{\varepsilon} \lambda}\right)\right], & \lambda \neq \frac{1}{2} \\
4 \eta \ln (1+\dot{\varepsilon} t), & \lambda=\frac{1}{2}\end{cases} \\
& \tau_{\mathrm{v} 22}=\tau_{\mathrm{v} 33}=-\frac{\eta}{\lambda} \exp \left(-\frac{1+\dot{\varepsilon} t}{\dot{\varepsilon}} \frac{\lambda+1}{\lambda}\right)\left[E i\left(\frac{1+\dot{\varepsilon} t}{\varepsilon} \frac{\lambda+1}{\lambda}\right)-E i\left(\frac{2 \lambda+1}{\dot{\varepsilon} \lambda}\right)\right] \\
& \tau_{\mathrm{v} 12}=\tau_{\mathrm{v} 13}=\tau_{\mathrm{v} 23}=0
\end{aligned}
$$

where $\tau_{\mathrm{v} i j}$ is the components of the viscoelastic stress tensor $\boldsymbol{\tau}_{\mathrm{v}}, E i$ is the exponential integral function defined by

$$
E i(z)=-\int_{-z}^{\infty} e^{-t} / t d t
$$

The components $\tau_{\text {sij }}$ of the strain-hardening stress tensor $\boldsymbol{\tau}_{\mathrm{s}}$ can be directly written from eq. (3) by noting that

$$
\mathbf{B}=\left(\begin{array}{ccc}
\alpha^{2} & 0 & 0 \\
0 & \alpha^{-1} & 0 \\
0 & 0 & \alpha^{-1}
\end{array}\right)
$$

under uniaxial tension, as

$$
\tau_{\mathrm{s} 11}-\tau_{\mathrm{s} 22}=-\frac{E J_{\mathrm{m}}\left(\alpha^{3}-1\right)}{3\left[2-\left(3+J_{\mathrm{m}}\right) \alpha+\alpha^{3}\right]} .
$$

Finally, the tensile true stress $\sigma_{\mathrm{t}}$ as a function of the tensile time $t$ under constant engineering strain rate $\dot{\varepsilon}$ can be expressed into explicit form by $\sigma_{\mathrm{t}}=\tau_{\mathrm{v} 11}-\tau_{\mathrm{v} 22}+\tau_{\mathrm{s} 11}-\tau_{\mathrm{s} 22}$ using the results listed above.

Note that our model is the same as the one used by Deplace et al. (Soft Matter, 2009, 5, 1440), but we use the exact solution for constant engineering strain rate in the present work, instead of an approximation by the one for constant true strain rate. 
Table S1. Detailed compositions of 2.1-7/3, 2.3-7/3 and 2.5-6/4 hydrogels.

\begin{tabular}{ccccc}
\hline Hydrogel & $\begin{array}{c}\text { Total concentration } \\
(\mathrm{mol} / \mathrm{L})\end{array}$ & $\begin{array}{c}\text { NaSS } \\
(\mathrm{mol} / \mathrm{L})\end{array}$ & $\begin{array}{c}\text { MPTC } \\
(\mathrm{mol} / \mathrm{L})\end{array}$ & $\begin{array}{c}\text { DMAEMA } \\
(\mathrm{mol} / \mathrm{L})\end{array}$ \\
\hline $2.1-7 / 3$ & 2.1 & 1.05 & 0.735 & 0.315 \\
$2.3-7 / 3$ & 2.3 & 1.15 & 0.805 & 0.345 \\
$2.5-6 / 4$ & 2.5 & 1.25 & 0.75 & 0.5 \\
\hline
\end{tabular}



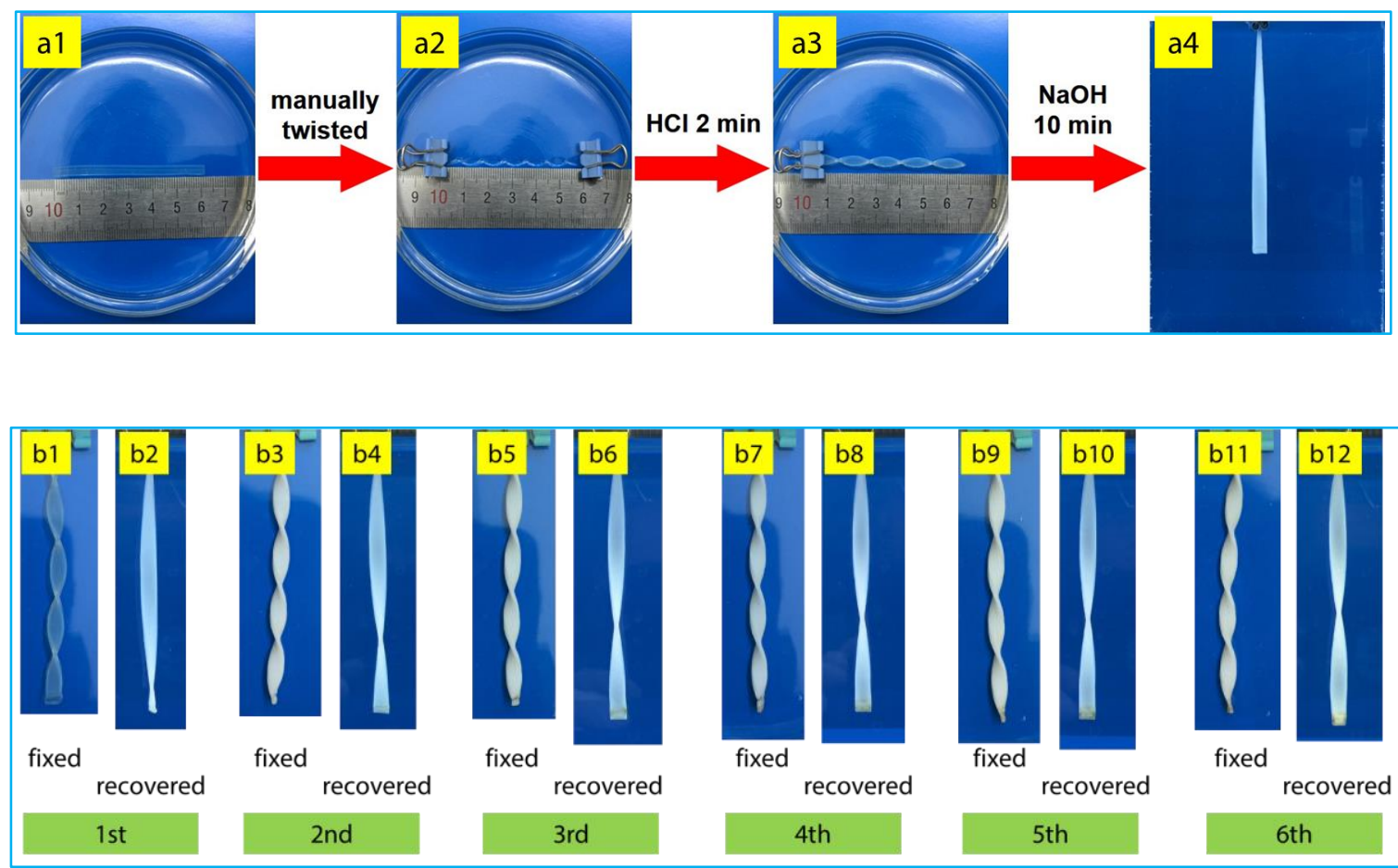

Figure S1. The shape-fixing and shape-recovery of the 2.1-7/3 hydrogel (a1-a4), and photos of the hydrogel during the first six shape memory cycles (b1-b12).

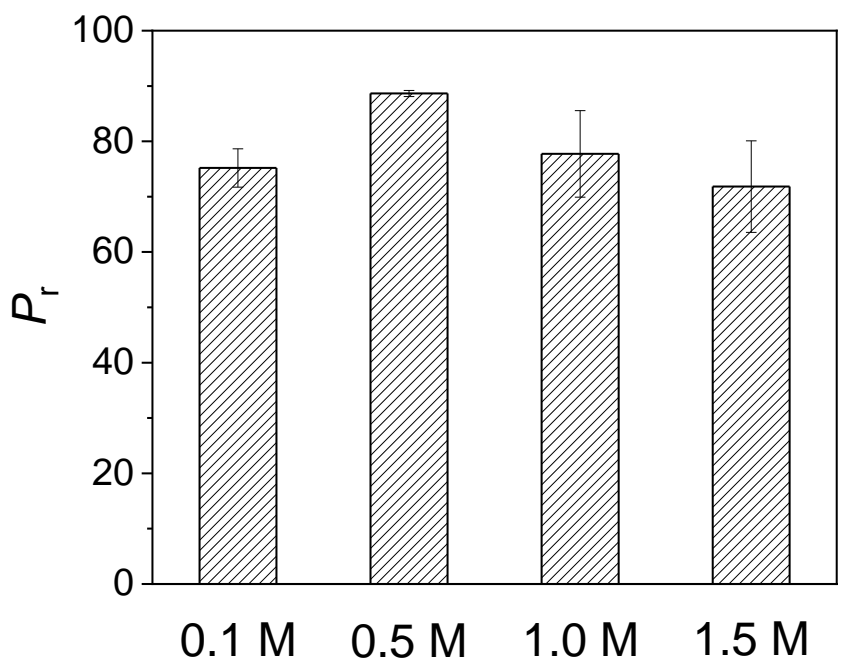

Figure S2. The influence of the concentration of $\mathrm{NaOH}$ on shape recovery percentage $P_{\mathrm{r}}$ of 2.1-7/3 hydrogel with fixed $\mathrm{HCl}$ concentration of $0.1 \mathrm{M}$. 

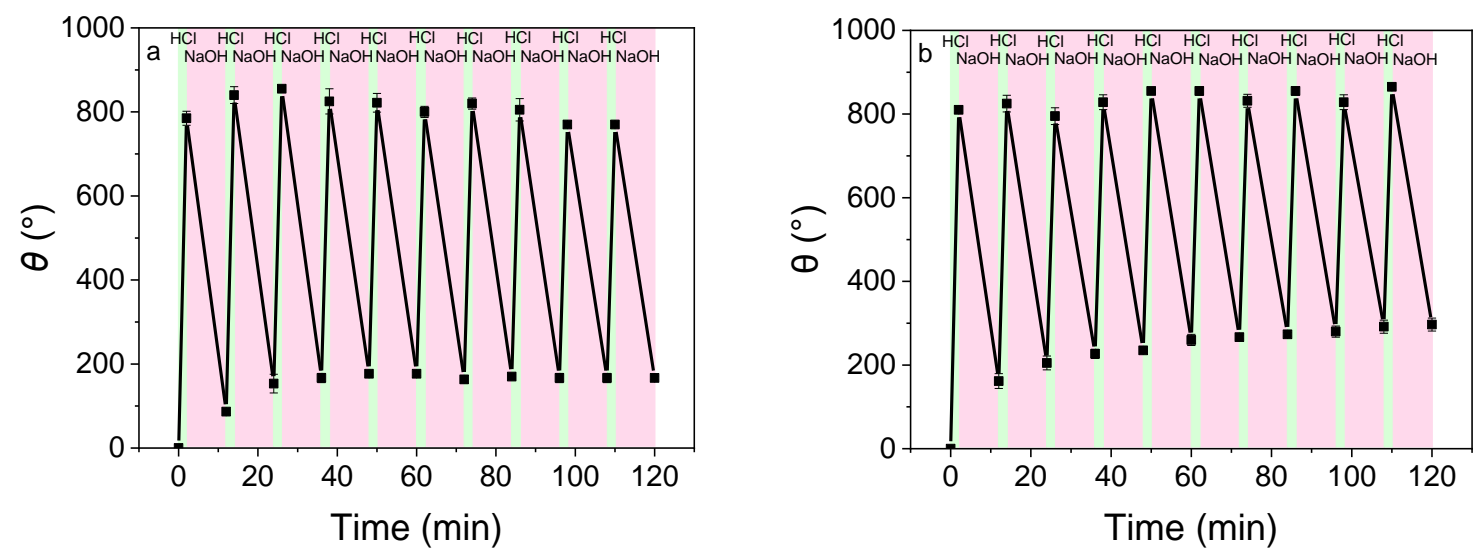

Figure S3. Shape-fixing and recovering cycles of (a) 2.5-6/4 and (b) 2.3-7/3 hydrogels; the as-prepared hydrogel strip was firstly twisted manually and fixed in $0.5 \mathrm{M} \mathrm{HCl}$ and then recovered to its original shape in $0.5 \mathrm{M} \mathrm{NaOH}$. The subsequent cycles were operated with the same steps.

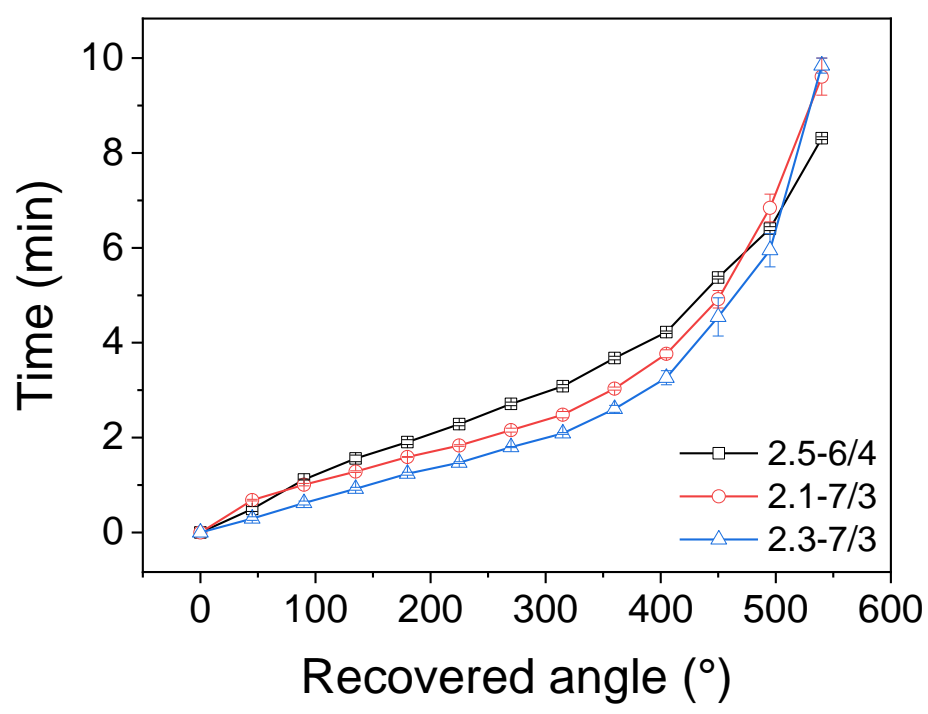

Figure S4. Plots of time needed for recovering to a certain angle for the $2.5-6 / 4,2.1-7 / 3$, and 2.3-7/3 hydrogels in $0.5 \mathrm{M} \mathrm{NaOH}$ for $10 \mathrm{~min}$. 

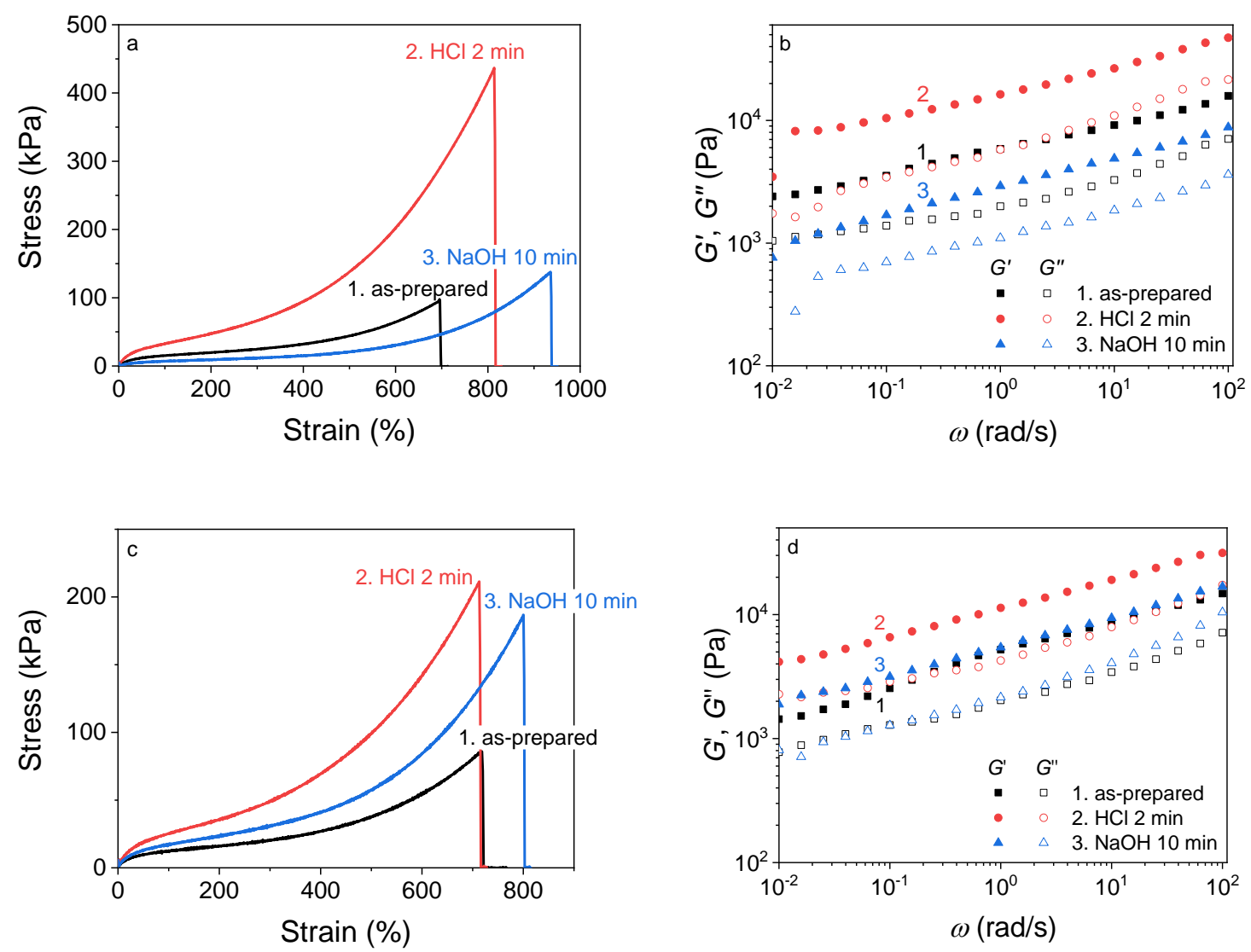

Figure S5. Tensile stress-strain curves and storage modulus $G^{\prime}$ and loss modulus $G^{\prime \prime}$ of 2.56/4 (a, b) and 2.3-7/3 (c, d) hydrogels after special immersion. 1. as-prepared hydrogel; 2. after immersed in $0.5 \mathrm{M} \mathrm{HCl}$ for $2 \mathrm{~min}$; 3. after immersed in $0.5 \mathrm{M} \mathrm{HCl}$ for 2 min and in $0.5 \mathrm{M}$ $\mathrm{NaOH}$ for $10 \mathrm{~min}$. 


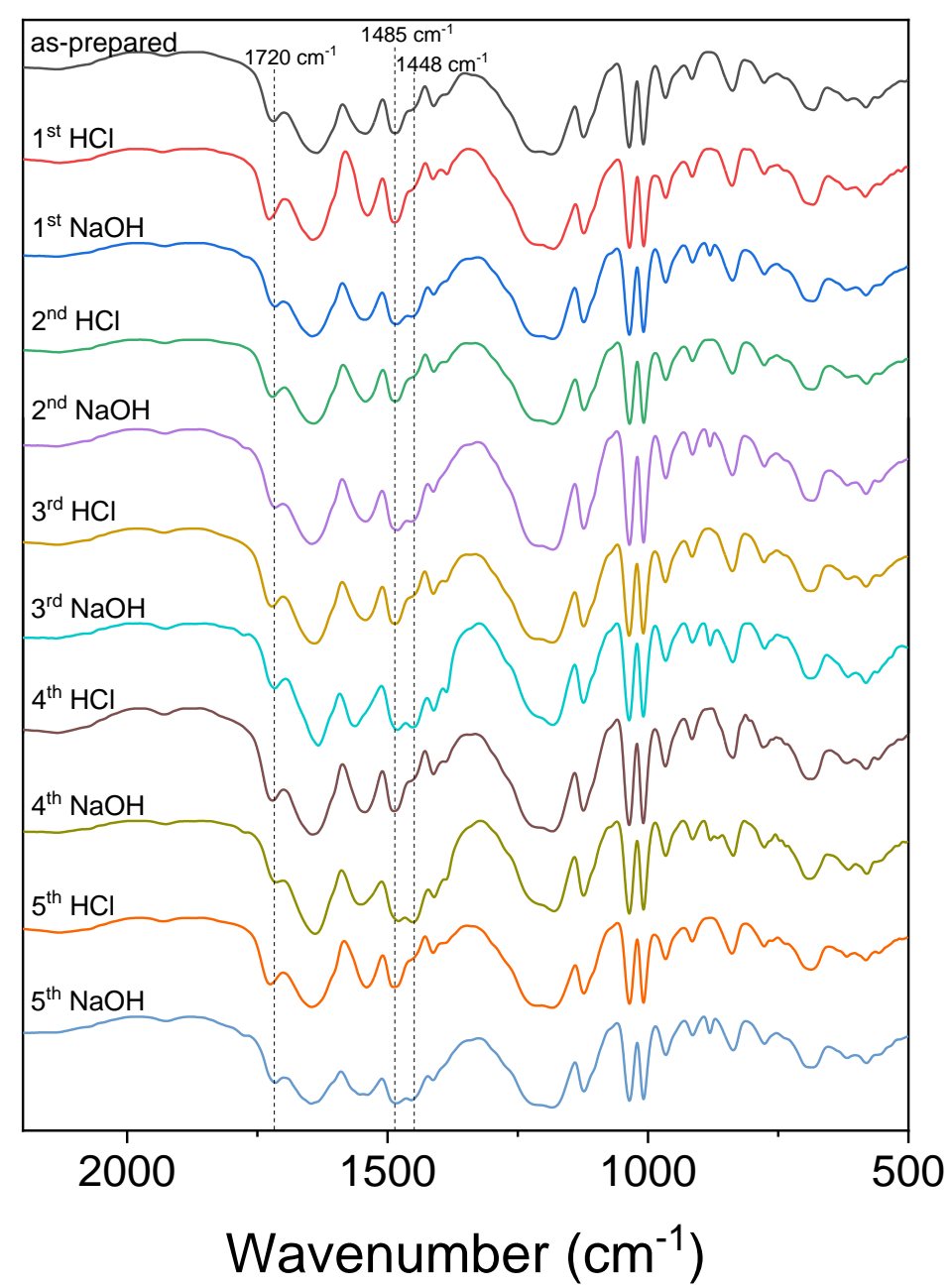

Figure S6. FTIR spectra of the 2.1-7/3 hydrogel during alternate immersion treatments: $1^{\text {st }}$ cycle: in $0.5 \mathrm{M} \mathrm{HCl}$ for $2 \mathrm{~min}$, in $0.5 \mathrm{M} \mathrm{NaOH}$ for 10 min after immersed in $0.5 \mathrm{M} \mathrm{HCl}$ for $2 \mathrm{~min}$, and then the following cycles. 


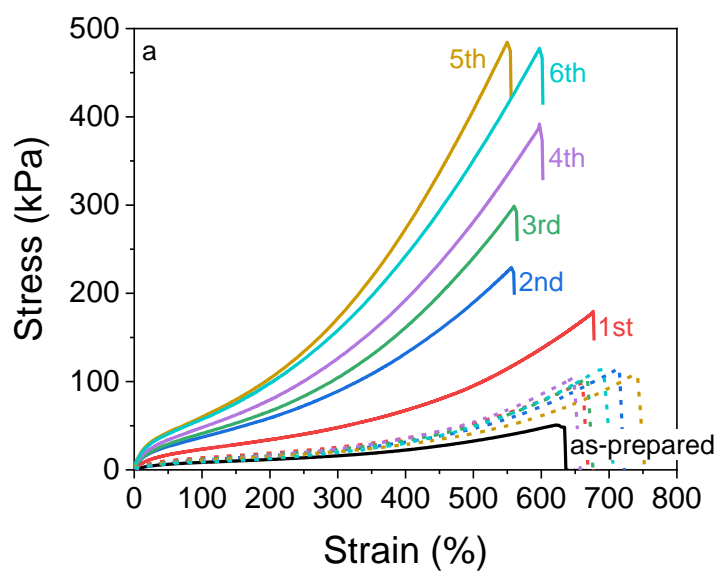

Figure S7. Stress-strain curves of the 2.1-7/3 hydrogel during the self-reinforcing process, the immersion treatments: in $0.5 \mathrm{M} \mathrm{HCl}$ for $2 \mathrm{~min}$ (solid line), in $0.5 \mathrm{M} \mathrm{NaOH}$ for $10 \mathrm{~min}$ after immersed in $0.5 \mathrm{M} \mathrm{HCl}$ for $2 \mathrm{~min}$ (dash line), numbers stand for the immersing cycle times.

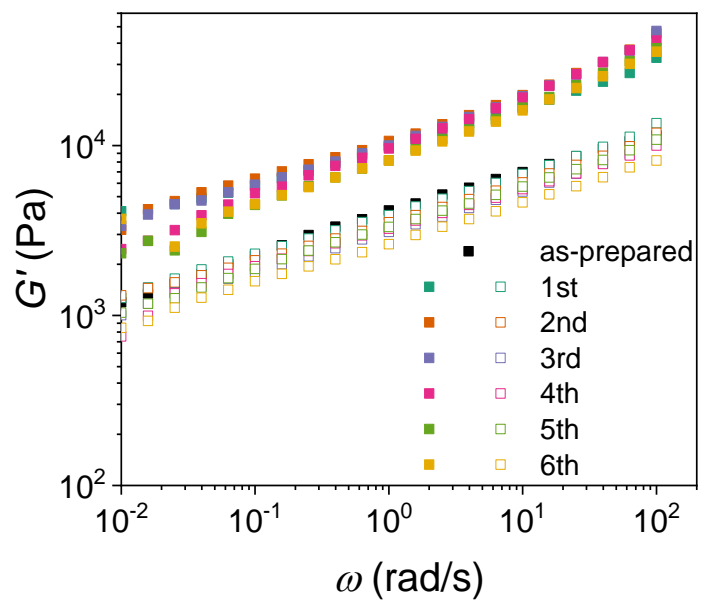

Figure S8. Storage modulus $G^{\prime}$ of the 2.1-7/3 hydrogel during the self-reinforcing process, the immersion treatments: in $0.5 \mathrm{M} \mathrm{HCl}$ for $2 \mathrm{~min}$ (solid symbols), in $0.5 \mathrm{M} \mathrm{NaOH}$ for $10 \mathrm{~min}$ after immersed in $0.5 \mathrm{M} \mathrm{HCl}$ for $2 \mathrm{~min}$ (open symbols), numbers stand for the immersing cycle times. 


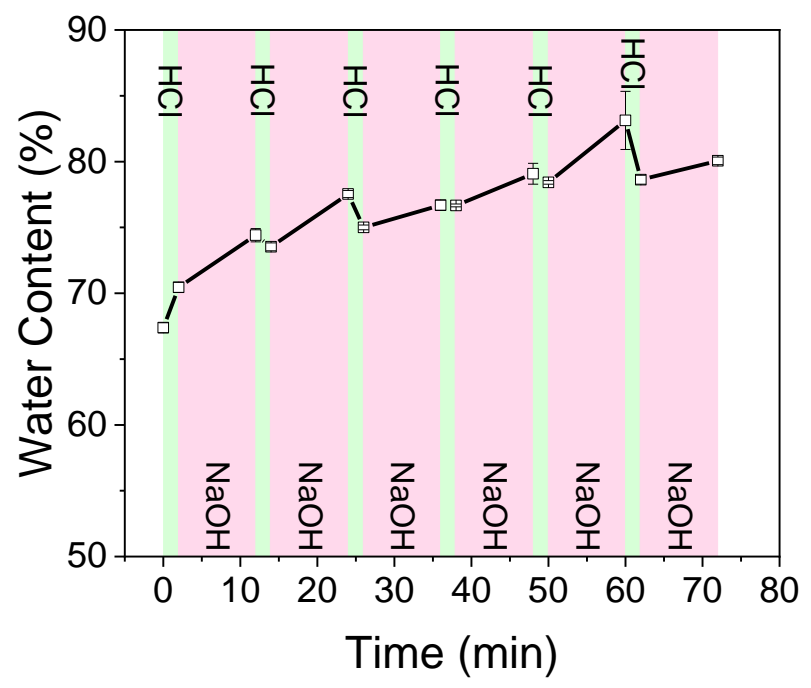

Figure S9. Water content of the 2.1-7/3 hydrogel during the self-reinforcing process.

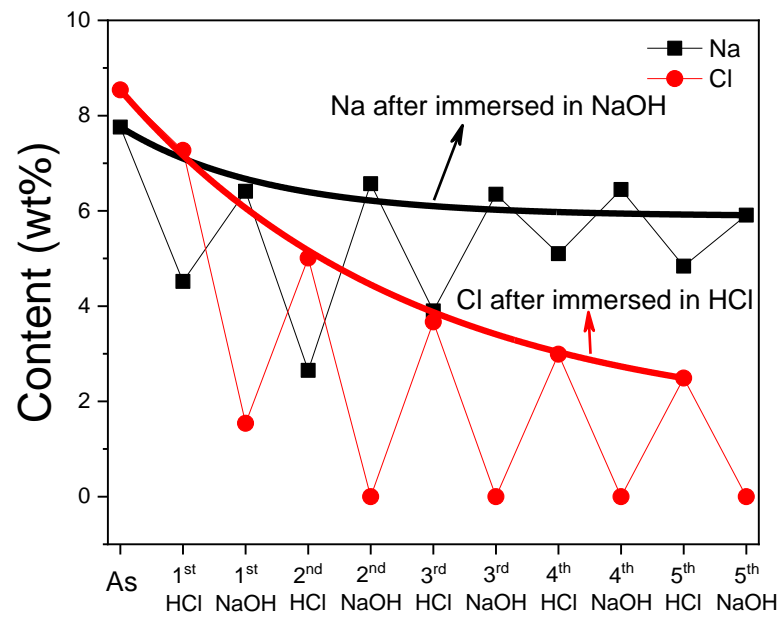

Figure S10. The content of $\mathrm{Na}$ and $\mathrm{Cl}$ elements on the 2.1-7/3 hydrogel surface during alternate immersion in $0.5 \mathrm{M} \mathrm{HCl}$ and $0.5 \mathrm{M} \mathrm{NaOH}$. As represents for the as-prepared hydrogel. 


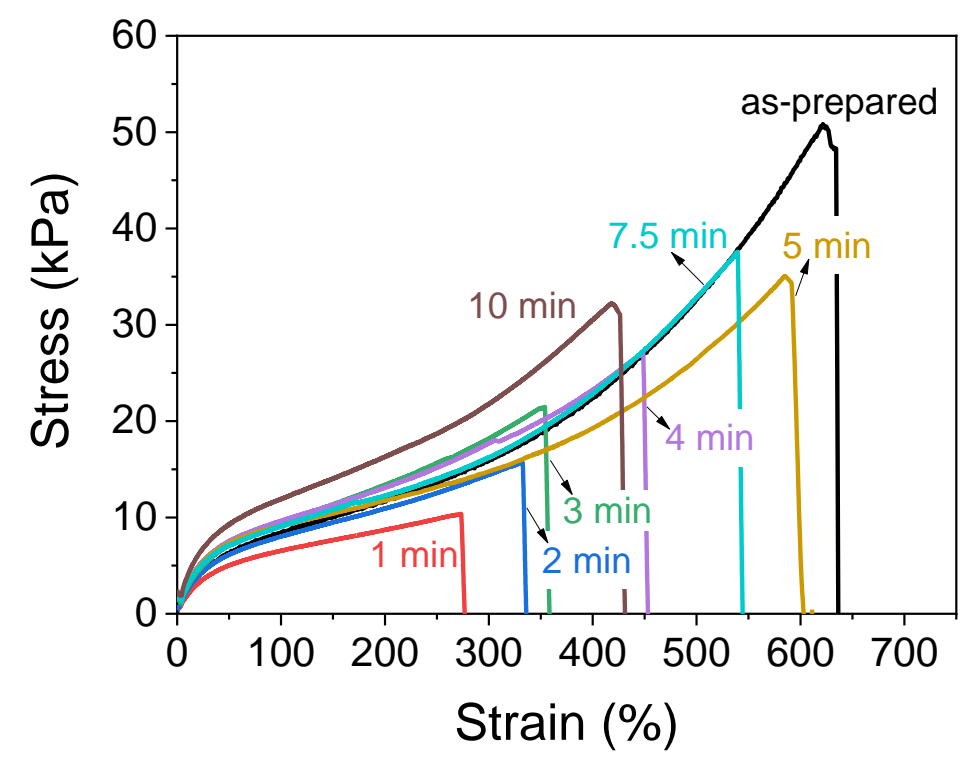

Figure S11. Stress-strain curves of the 2.1-7/3 hydrogel after healing for indicated time.

\section{Supporting movies:}

Movie M1. Opening of the 2.1-7/3 hydrogel box in $0.5 \mathrm{M} \mathrm{NaOH}$.

Movie M2. Blooming up of the 2.1-7/3 hydrogel flower in $0.5 \mathrm{M} \mathrm{NaOH}$.

Movie M3. Red liquid flowing in the healed 2.1-7/3 hydrogel tube.

Movie M4. The brightness changes of the LED lamp connected with the as-prepared and selfhealed 2.1-7/3 hydrogel under stretching and recovering. 